\title{
¿Todo tiempo pasado fue mejor? El turismo frente a los desafíos de la nueva normalidad
}

\section{All Times Gone by Were Far Better? Tourism Facing the Challenges of the New Normality}

\section{Vanesa Castello}

Vanesa Castello es docente e investigadora de la Escuela de Relaciones Internacionales, Facultad de Ciencia Política y Relaciones Internacionales, Universidad Nacional de Rosario, Argentina.

E-mail: vanesa.castello@fcpolit.unr.edu.ar

\section{resumen}

Enfrentarnos a la pandemia del Coronavirus (Covid-19) nos posiciona ante la primera gran crisis sanitaria del último siglo, con un impacto significativo en los niveles social, económico, político y cultural que ponen de manifiesto las críticas a la globalización que ya estaban sobre la mesa de discusión.

Si bien el turismo ha sabido recuperarse de otras crisis ocurridas, por primera vez la actual pandemia provocó una total interrupción en los flujos de viajeros tanto en el interior como entre los países. La pandemia -y el consecuente cierre de fronteras- ha acelerado y profundizado el cuestionamiento a la manera en que los países venían gestionando el turismo, sobre todo en función de los efectos negativos sobre las comunidades de destino y sus patrimonios, así como sobre el ambiente.

El turismo sostenible se erige como estructurador de la recuperación del turismo a nivel mundial.

\section{summary}

Facing the Coronavirus (Covid-19) pandemic positions us in front of the first major health crisis of the last century with a significant impact on the social, economic, political and cultural levels, bringing out the critics towards globalization that were already on the discussion table.

Although tourism has managed to recover from other crises that have occurred, for the first time the current pandemic caused a total interruption in the flow of travelers both inland and between countries. The pandemic -and the consequent closure of borders-, has accelerated and deepened the questioning of the way countries have been managing tourism, especially based on the negative effects on destination communities and their heritage, as well as on the environment. Sustainable tourism stands as a structuring factor for the recovery of tourism worldwide. palabras clave

turismo / Covid-19 / sostenibilidad

\section{keywords}

tourism / Covid-19 / sustainability 


\section{Introducción}

Enfrentarnos a la pandemia del Coronavirus (Covid-19) nos posiciona frente a la primera gran crisis sanitaria del último siglo, con un impacto significativo en los niveles social, económico, político y cultural que ponen de manifiesto los cuestionamientos a la globalización que ya estaban sobre la mesa de discusión. Al presentar una alta tasa de contagios, con cifras que superan los 10 millones de casos confirmados en el mundo y más de 500 mil muertes en solo medio año, los cierres de las fronteras han afectado directamente al sector turístico, referente por excelencia de la dinámica de la sociedad global.

Si bien el turismo ha sabido recuperarse, o bien era hasta la fecha un sector que parecía casi inmune a diversas crisis ocurridas - desde la aparición del SARS en 2003, hasta la debacle económica financiera de 2008-, por primera vez la actual pandemia provocó que un $75 \%$ de los países adhirieran a una total interrupción en los flujos de viajeros tanto en el interior como entre los países. Esto provocó una caída del 97\% en las llegadas de turistas internacionales. La crisis por el Covid-19 ha acelerado y profundizado el cuestionamiento a la manera en que los países venían gestionando el turismo, sobre todo en función de los efectos negativos sobre las comunidades de destino y sus patrimonios, así como sobre el ambiente.

Esta situación nos presenta el desafío de pensar cómo evolucionará y se desarrollará la actividad una vez que puedan controlarse los contagios, fundamentalmente en función de las medidas que los países tomen a la hora de gestionar la apertura de sus fronteras al movimiento de viajeros, así como al contemplar las dispares realidades entre países.

\section{El sector turístico frente a un contexto incierto, pero de oportunidades}

El turismo ha sido definido por diversos autores y desde diferentes perspectivas. La definición acordada en el marco internacional multilateral es la ofrecida por la Organización Mundial del Turismo (OMT), que indica que el turismo integra las actividades que realizan las personas durante sus viajes y estancias en lugares distintos a su entorno habitual durante un período de tiempo inferior a un año, con fines de ocio, negocios u otros. Podemos afirmar que el turismo es, entonces, una práctica social protagonizada por sujetos sociales, los turistas.

Sin embargo, esta definición guarda silencio y provoca un "bloqueo de su conceptualización" (Bertoncello, 2002) en relación con otros actores involucrados, como los agentes económicos o las comunidades de destino y origen. Desaprovecha, así, la oportunidad de analizar el fenómeno en el marco de las realidades sociales, culturales y económicas específicas dentro de las cuales tiene lugar. Sin incorporar toda la gama de actores, contextos y realidades involucrados, dicha lectura resulta incapaz de dar cuenta de la significancia que el turismo concentra, en particular en contextos tan especiales e inciertos como los que la pandemia de Covid-19 nos presenta.

Resulta interesante, entonces, el planteo de Urry (1996), para quien el turismo debe pensarse no solo desde el lugar de destino, sino también desde la sociedad de origen. De este modo, los lugares de origen y destino son articulados por los turis- 
tas, que llevan la actividad dentro del marco de sus prácticas cotidianas, inscriptas a su vez en prácticas sociales más generales. Por lo tanto, debemos reparar en que el análisis de la elección de una determinada práctica turística debe estudiarse dentro de contextos sociales y espaciales específicos.

Las restricciones a la circulación impuestas por la mayoría de los países, a partir de las recomendaciones establecidas por la Organización Mundial de la Salud, significaron que las proyecciones establecidas para el sector en el año 2020, de un crecimiento en los viajes de entre $3 \%$ y $4 \%$ a nivel mundial, quedaran evidentemente no solo desactualizadas, sino que resultaron inadecuadas para reflejar la realidad que la industria enfrentaría. Frente a la dinámica incierta que la pandemia ha presentado, y las consecuentes acciones que los gobiernos desarrollaron para enfrentarla, las nuevas proyecciones establecen que el turismo internacional podría caer, entonces, entre un $60 \%$ y $80 \%$ este año (OMT, 2020a). Se trata de una realidad sin precedentes, si tenemos en cuenta que la caída de la actividad en otras ocasiones ha promediado un $4 \%$, sumado a que las pequeñas y medianas empresas representan el $80 \%$ del sector en todo el mundo. Resulta evidente que urge intentar minimizar los efectos sociales que la crisis efectivamente dejará.

De esta manera, el mundo del turismo se encuentra en medio de una crisis inédita por sus características. La pandemia ha causado graves perjuicios para el sector a lo largo del planeta, aunque parece que, para algunas regiones, los efectos y la futura recuperación se presentan más agudos y complicados que en otras. En particular, resultará más difícil para aquellos países en desarrollo cuyas economías son altamente dependientes o se basan casi de manera exclusiva en los ingresos que genera el tursimo, y que presentan, en muchos de estos casos, adicionales debilidades estructurales en sus sistemas económicos, sociales y sanitarios.

Es un hecho que, desde mediados del siglo XX, pero fundamentalmente en estas últimas décadas, en los países en desarrollo, el sector turístico exhibía un rápido crecimiento, y se había convertido en un actor protagonista de las economías de los países. Las estadísticas demostraban, entre otras cosas, que el turismo representaba más de un $10 \%$ del PBI mundial, uno de cada diez empleos y el $30 \%$ de las exportaciones mundiales de servicios. Asimismo, según la OMT (2020b), se estaba produciendo una expansión geográfica y una diversificación sustancial de los destinos elegidos, donde los países en desarrollo eran aquellos que lograban el mayor crecimiento. En este sentido, en los últimos años, las llegadas de turistas a estos países alcanzaron a representar el $46 \%$ del total de los flujos internacionales. Esto significa la primera o segunda fuente de divisas para 20 de los 48 países menos avanzados. Estos datos, claro está, dan cuenta de la situación pre-pandemia.

Entre tanto, grandes segmentos de la actividad turística se desarrollaban dentro de un paradigma de crecimiento indefectible e inevitable. El hecho de erigirse como un importante motor de desarrollo económico, con la capacidad de generar divisas para los países, redistribuir el ingreso dentro del territorio nacional, dinamizar las economías regionales, crear puestos de trabajo y promover la ejecución de infraestructuras en muchos destinos alrededor del mundo, fundamentaba la defensa de su evolución imparable (Brohman, 1996; Gibson, 1993). Desde esta 
perspectiva, el turismo favorecía el desarrollo económico de los países en toda su cadena de valor. Facilitaba, asimismo, que se generara -como consecuencia lógica de este desarrollo- la mejora de la apreciación de la cultura e identidad local, que se contribuyera a la protección de áreas naturales, al mantenimiento de áreas urbanas y sitios históricos, etcétera (Conti y Cravero Igarza, 2010).

Si bien no exclusivamente, pero a partir de las desalentadoras e inciertas proyecciones mencionadas para la actividad en 2020 y principios del próximo año, lo que la crisis por el Covid-19 vino a poner sobre la mesa de discusión son temas que ya preocupaban a la comunidad internacional. La manera en que se gestionaba el turismo, el agotamiento de la llamada capacidad de carga de los destinos y de sus patrimonios naturales y culturales, la desatención sobre las comunidades locales y las repercusiones ambientales son temas que integraban la agenda de aquellas miradas propias de lo que Jafari (2005) llamó la "plataforma precautoria". Es decir, la crisis sanitaria profundizó el agotamiento que el modelo de turismo masivo transitaba, fundamentalmente por los efectos devastadores del overtourism: estacionalidad en el empleo, desarrollo desequilibrado entre regiones, dependencia exterior, destrucción de recursos, mercantilización de comunidades y de su cultura, religión $\mathrm{y}$ artes, conflictos en las comunidades de acogida, entre otras. Como bien lo indica Malacalza (2020), la pandemia, o esta situación límite, aceleró las tendencias hacia la crisis de la globalización, y el turismo no ha sido la excepción.

En esta línea, algunos expertos hablaban de neo-colonización turística, ya que se dirigía una gran parte de las inversiones públicas hacia la satisfacción del turista internacional y de las grandes empresas transnacionales turísticas, en detrimento de la población local y de sus necesidades. Al mismo tiempo, se privatizaban grandes extensiones de tierras de cultivo y de playas. Hasta un $75 \%$ del dinero que pagaban los turistas durante su estancia retornaba al país de origen de la empresa comercializadora, con sede generalmente en países del Norte. Esto dejaba unos beneficios marginales en el país visitado (SODEPAZ, s.f.).

\section{La sostenibilidad como la nueva normalidad. EI desafío global y regional}

Pese a que la apertura de la actividad en algunos países europeos y asiáticos se presenta promisoria para el futuro de los viajes -no sin limitaciones aún-, los cambios que inexorablemente la pandemia ha producido, y aún producirá, en la manera en la que la gente realiza turismo podrían llegar a presentar algunas dificultades para los países de Latinoamérica y el Caribe, incluso con la total apertura de fronteras para el tránsito de personas con fines turísticos. Las referencias a cuestiones de seguridad y sanitarias se erigirán probablemente como factores determinantes a la hora de la elección que los viajeros establecerán como prioridades. Estos son elementos sobre los que estos Estados deberán prestar atención. Sin embargo, para estos países, ciertas debilidades estructurales se imponen al tiempo que les urge recomponer un sector del cual muchos de ellos son altamente dependientes.

Lo cierto es que recomendaciones del Foro Económico Mundial (2020) a los países de Latinoamérica y el Caribe establecen dedicar este tiempo a revisar sus proyectos de promoción turística a los fines de forjar un mejor sector en el futuro. 
Entre ellas, se despliega un abanico de temas que van desde la mejora de la capacidad sanitaria hasta la extensión del uso de herramientas TIC, o tecnologías de la información y la comunicación, a la hora de contratar y desarrollar viajes. Se espera que ambas cuestiones cobren mayor importancia en contextos donde las interacciones interpersonales se vean limitadas.

Diversas organizaciones, especialistas y entes dedicados al turismo coinciden en que, más allá de las medidas locales, es indispensable desarrollar estándares globales. En este marco, la OMT creó y dirige un Comité Mundial de Crisis Turística para coordinar una guía global de recuperación y normalización de la situación. Dicho comité está conformado por representantes de sus Estados y Miembros Afiliados, así como de la Organización Mundial de la Salud, la Organización de Aviación Civil Internacional y la Organización Marítima Internacional. El sector privado está representado por el Consejo Internacional de Aeropuertos, la Asociación Internacional de Líneas de Crucero, la Asociación de Transporte Aéreo Internacional y el Consejo Mundial de Viajes y Turismo. El objetivo es garantizar una respuesta coordinada y efectiva por parte de los sectores público y privado. Por otro lado, se busca fomentar la cooperación internacional para subrayar una respuesta unida y minimizar los efectos económicos y el costo social de la pandemia. ${ }^{1}$

El confinamiento ha ralentizado aún más los proyectos infraestructurales en Latinoamérica y el Caribe, y parecería que la mera apertura internacional del destino no será el único factor por considerar. El desafío es pensar cómo los países latinoamericanos y caribeños estarán en condiciones de alcanzar esos estándares -que, por ahora, se dan en términos de recomendaciones- que, en la mayoría de los casos, parecen apropiados o factibles para países desarrollados. De igual manera, es preciso considerar que cualquier restricción a los viajes o introducción de nuevas medidas y políticas, así como nuevas medidas de distanciamiento social, deberían basarse en evidencia, para no transformarse en medidas discriminatorias.

Sin embargo, para aportar optimismo a lo hasta aquí relatado, debemos rescatar que los amplios recursos naturales de Latinoamérica y el Caribe serán claves para su recuperación. Diversas encuestas dan cuenta de que los turistas quieren volver a viajar, pero que recurrirán a experiencias más personalizadas en ambientes fuera de las grandes ciudades y más cercanas a la naturaleza. En función de la manera dispar en que los países están abriendo sus fronteras -en términos de países aceptados, normativa a aplicarse para los turistas, etcétera-, lo más probable es que primero se reactive el turismo local, luego el regional -las llamadas "burbujas" o corredores turísticos- $y$, por último, el internacional.

En este marco, es indudable que el turismo tendrá gran relevancia como una herramienta que contribuirá a la recuperación económica de muchos de nuestros países. Sin embargo, esto será así siempre y cuando contemplemos que esa recuperación se viabilizará en tanto el turismo se gestione centrándose prioritariamente en la atenuación de la pobreza, para beneficiar directamente a los grupos más desprotegidos, mediante el empleo de la población local en empresas turísticas y en el suministro de bienes y servicios a los turistas, en la gestión de pequeñas empresas 
y empresas comunitarias, en el cuidado del ambiente y el respeto a las poblaciones locales y su patrimonio.

Durante años se ha teorizado alrededor del concepto de turismo sostenible, es decir, aquel que ha de ser soportable ecológicamente a largo plazo, viable económicamente y equitativo desde una perspectiva ética y social para las comunidades locales. La actual pandemia nos presenta una circunstancia histórica para efectivamente concentrarnos en ese concepto y trabajar sobre los temas que la gestión del turismo, tal cual lo conocemos, presenta como deuda. El decrecimiento del turismo, que no equivale a una desaceleración descontrolada, ni al "antiturismo", supone la oportunidad para que la actividad se organice y se practique de una manera distinta y a escala diferente, para evitar muchos de los impactos negativos asignados al sector.

En este marco, el Programa de Turismo Sostenible de One Planet, liderado por la OMT, asumió el compromiso de encarar acciones para la preparación, la promoción y el escalonamiento de prácticas de consumo y producción sostenibles que impulsen un uso eficiente de los recursos naturales. A su vez, apuesta a que se generen menos residuos y se afronten los retos del cambio climático y la pérdida de biodiversidad. One Planet recomienda seis líneas de acción para guiar un turismo sostenible: 1) recuperación para las personas, el planeta y la prosperidad; 2) salud pública; 3) inclusión social; 4) conservación de la biodiversidad; 5) acción climática; y 6) economía circular, gobernanza y finanzas, bajo los conceptos de reducir, reutilizar, restaurar y uso eficiente de la energía y el agua.

Para ello, es indispensable que exista un genuino interés en un Estado presente, que sea capaz de impulsar la actividad tanto en las crisis como en los tiempos de prosperidad, así como el impulso y retorno del turismo a la agenda de la cooperación y la sostenibilidad. Resulta prioritario trabajar sobre la capacidad de carga de los destinos turísticos y atractivos para velar por el cuidado del ambiente y de las comunidades, pero también es indispensable el trabajo en torno a la difusión y promoción de actividades turísticas que, en este sentido, son cruciales para la capacidad de recuperación del turismo a largo plazo en la región.

Finalmente, cabe recordar que el turismo se incluye como parte de tres Objetivos de Desarrollo Sostenible (ODS): el Objetivo 8, sobre crecimiento económico y empleo; el Objetivo 12, sobre producción y consumo sostenibles; y el Objetivo 14 , sobre vida submarina. Sin embargo, el turismo puede y debe desempeñar un papel importante en las soluciones que se encuentren en el marco de todos y cada uno de los 17 ODS, y conformar así el escenario ideal para que el sector reflexione sobre su papel y su contribución verdadera como herramienta de un desarrollo integral para todos los países.

\section{A modo de reflexión}

Si bien el cuestionamiento al modelo de gestión del turismo masivo estaba presente en los debates pre-pandemia, la crisis sanitaria aceleró la necesidad de efectivizar un cambio. Este cambio requiere que se consideren diversas cuestiones, como un rol más activo del Estado en la planificación de políticas para el turismo, 
la generación de acciones de cooperación entre el sector público y privado, la consideración de las realidades de países como los de Latinoamérica y el Caribe-que dependen en gran medida de los ingresos que el sector genera-, la voz y respeto a las comunidades locales receptoras, y la adecuación a la capacidad de carga de destinos y sus patrimonios culturales y naturales.

El deseo y necesidad de reactivar el turismo no nos debe hacer retornar a prácticas sin planificación, que probaron ser dañinas para el ambiente y para el futuro de la actividad a largo plazo. Los descensos del turismo brindan a los responsables políticos y líderes empresariales de la región la oportunidad de revisar sus prácticas y políticas turísticas, especialmente en materia de infraestructuras y desarrollo turístico sostenible. Nos urge una medición del rendimiento del turismo más allá de lo estrictamente económico.

La pandemia aceleró estos debates y el alcance a una solución basada en un turismo sostenible. En este marco, la recuperación del sector turístico debe apostar a que lo mejor está por venir. El objetivo es que una reducción voluntaria y planificada en el flujo y concentración de turistas tal cual los conocíamos permita, sin dejar de promover la generación de empleo e ingresos para los países, maximizar los beneficios para las comunidades locales y el medioambiente.

\section{Referencias}

1. Hasta el momento, se han emitido recomendaciones en torno a básicamente tres grupos de temas: supervivencia de la industria con medidas de protección, recuperación de la confianza, y preparación para el futuro. Las prioridades incluyen crear nuevos puestos de trabajo, proteger la salud, recuperar la confianza y trabajar de manera conjunta (OMT, 2020c). Asimismo, el Consejo Mundial de Viajes y Turismo, que incluye a las principales compañías de viajes y turismo del mundo, presentó lo que llaman una serie de protocolos de recuperación efectivos mediante el desarrollo de planes de acción significativos que optimicen los esfuerzos de recuperación en todo el sector.

\section{Bibliografía}

Bertoncello, R. (2002). Turismo y territorio. Otras prácticas, otras miradas. Aportes y Transferencias, 6. (2), 29-50. Disponible en línea: http://nulan.mdp.edu.ar/259/

Brohman, J. (1996). New Directions in Tourism for Third World Development. Annals of Tourism Research, na23, 48-70. Disponible en: https://doi.org/10.1016/0160-7383(95)00043-7

Conti, A. y Cravero Igarza, S. (2010). Patrimonio, comunidad local y turismo. La necesidad de planificación para el desarrollo sostenible. Notas en Turismo y Economía, Instituto de Investigaciones Económicas, año I, nro. I., 8-31. Disponible en: http://sedici.unlp.edu.ar/handle/10915/15769 Consultado $\underline{\text { marzo } 2020}$

Foro Económico Mundial (2020). El panorama de la competitividad de los viajes y el turismo en América Latina y el Caribe: evaluación de oportunidades y desafíos regionales en el contexto de COVID19. Disponible en: https://www.weforum.org/reports/latin-america-and-caribbean-travel-tourismcompetitiveness-landscape-report-in-the-context-of-covid-19

Gibson, L. J. (1993). The Potential for Tourism Development in Nonmetropolitan Areas. En D. L. Barkley (ed.), Economic Adaptation: Alternatives for Nonmetropolitan Areas, pp. 145-164. Boulder, US: Westview Press.

Jafari, J. (2005). El turismo como disciplina científica. Política y Sociedad, 42(1), 39-56. Disponible en: https://revistas.ucm.es/index.php/POSO/article/view/POSO0505130039A

Malacalza, B. (2020). ¿Por qué es urgente la cooperación internacional? En El País (30/03/2020). Dis- 
ponible en: https://elpais.com/elpais/2020/03/26/planeta futuro/15852333726697773.html

OMT (2020a). La OMT lanza un llamamiento a la acción para la mitigación del impacto de la COVID19 en el turismo y la recuperación del sector. Disponible en: https://www.unwto.org/es/news/apoyoal-empleo-y-a-la-economia-a-traves-de-los-viajes-y-el-turismo

OMT (2020b). Turismo en Iberoamérica. Creando oportunidades para todos. Disponible en: https:// www.e-unwto.org/doi/book/10.18111/9789284421497

OMT (2020c). Apoyo al empleo y a la economía a través de los viajes y el turismo. Disponible en: https://webunwto.s3.eu-west-1.amazonaws.com/s3fs-public/2020-04/COVID19 Recommendations ES.pdf

Sodepaz (s/f). Turismo y Desarrollo: Experiencias desde la Cooperación Internacional. Proyecto: Turismo, Desarrollo y Sostenibilidad: el Turismo como herramienta de consumo responsable y transformación social. Madrid, España. Disponible en: https://sodepaz.org/images/cuadernos/turismo_y_cooperacion.pdf

Urry, J. (1996). O olhar do turista. Lazer e viagens ñas sociedades contemporáneas. São Paulo, Brasil: SESC y Studio Nobel.

-WORLD TRAVEL \& TOURISM COUNCIL (2020), "Viajes seguros": protocolos globales y sello para la nueva normalidad. Disponible en línea: https://wttc.org/COVID-19/Safe-Travels-Global-Protocols-Stamp Consultado julio 2020

Vanesa Castello, "¿Todo tiempo pasado fue mejor? El turismo frente a los desafíos de la nueva normalidad". Revista Temas y Debates. ISSN 1666-0714, año 24, número especial, julio-diciembre 2020, pp. 439-446. 